\title{
ESPECIES DE ERETMOCERUS HALDEMAN (HYMENOPTERA: APHELINIDAE) DE MÉXICO CON MAZA ANTENAL CORTA, CLAVE Y DESCRIPCIÓN DE UNA NUEVA ESPECIE
}

\author{
Svetlana N. Myartseva, Enrique Ruiz-Cancino \& Juana María \\ Coronado-Blanco \\ División de Estudios de Postgrado e Investigación, Facultad de Ingeniería y Ciencias, Universidad \\ Autónoma de Tamaulipas, 87149 Cd. Victoria, Tamaulipas, México. <smyartse@uat.edu.mx>; \\ <eruiz@uat.edu.mx>; <jmcoronado@uat.edu.mx>
}

Myartseva, S. N., E. Ruiz-Cancino \& J. M. Coronado-Blanco. 2011. Especies de Eretmocerus Haldeman (Hymenoptera: Aphelinidae) de México con maza antenal corta, clave y descripción de una nueva especie. Acta Zoológica Mexicana (n. s.), 27(3): 583-590.

RESUMEN. Se incluye una breve revisión y una clave para identificar las especies mexicanas de Eretmocerus con maza antenal corta. Se describe Eretmocerus clavator Myartseva, sp. nov., del Estado de Jalisco, México.

Palabras clave: Hymenoptera, Aphelinidae, Eretmocerus, México.

Myartseva, S. N., E. Ruiz-Cancino \& J. M. Coronado-Blanco. 2011. Species of Eretmocerus Haldeman (Hymenoptera: Aphelinidae) from Mexico with short club, key and description of a new species. Acta Zoológica Mexicana (n. s.), 27(3): 583-590.

ABSTRACT. A short review and a key to identify the Mexican species of Eretmocerus with short antennal club are given. Eretmocerus clavator Myartseva, sp. n., from the State of Jalisco, Mexico, is described.

Key words: Hymenoptera, Aphelinidae, Eretmocerus, México.

\section{INTRODUCCIÓN}

El género Eretmocerus Haldeman tiene una distribución cosmopolita y comprende 74 especies descritas (Noyes 2003). Varias especies son muy importantes en el control biológico de mosquitas blancas (Homoptera: Aleyrodidae), las que pueden ser plagas agrícolas muy serias. Hasta enero del 2011, se conocían 20 especies de Eretmocerus en México (datos de los autores, no publicados). Tres especies se introdujeron a la República Mexicana para el control de la mosquita blanca Bemisia tabaci (Genna-

Recibido: 14/01/2011; aceptado: 09/05/2011. 
dius): Eretmocerus emiratus Zolnerowich \& Rose, E. eremicus Rose \& Zolnerowich y E. mundus Mercet (Noyes, 2003). Otras cinco especies del mismo género se han encontrado en México como enemigos naturales de Bemisia tabaci, incluyendo una nueva especie para la ciencia descrita por Myartseva (2006) y seis especies de Eretmocerus se encontraron como parasitoides de la mosquita blanca lanuda de los cítricos Aleurothrixus floccosus (Maskell), incluyendo otra nueva especie (Myartseva \& Coronado-Blanco 2007).

Considerando que la correcta identificación de las especies es un factor importante en cualquier programa de control y que en México no existe una clave para separar las especies de Eretmocerus presentes, se planteó el presente trabajo con los objetivos de realizar una revisión breve de las especies mexicanas con maza antenal corta, una clave para separar dichas especies y elaborar la descripción de una nueva especie de Eretmocerus.

\section{MATERIAL Y MÉTODOS}

Los especímenes de Eretmocerus se colectaron, criaron y preservaron usando la metodología propuesta por Noyes (1982). Al emerger de sus hospederos o al ser colectados con red, los parasitoides se atraparon con aspirador y se transfirieron a viales con alcohol 70\% para su preservación. Posteriormente, algunos especímenes se disectaron y montaron en laminillas en bálsamo de Canadá.

Para la identificación de las especies de Eretmocerus se utilizaron las claves de Hayat (1998), Rose (2000) y las de Myartseva \& Coronado-Blanco (2007), además de las descripciones originales. Se utilizaron criterios morfológicos, basados principalmente en algunas partes de la cabeza y el cuerpo, de acuerdo con Rose \& Zolnerowich (1997) y Zolnerowich \& Rose (1998): configuración de los segmentos antenales, proporciones relativas de los segmentos antenales, patrón de setas en el lóbulo medio del mesoescudo, proporción de la longitud y la anchura del ala anterior, longitud relativa del fleco marginal y de las venas de las alas anteriores, número de setas en la base y entre la vena marginal y la línea calva en las alas anteriores, longitud relativa de la tibia media y el basitarso de la pata media, longitud relativa del ovipositor, de la maza antenal y la tibia media.

El holotipo de la nueva especie se depositará en el Entomological Research Museum, University of California-Riverside (UCRC), USA.

\section{RESULTADOS Y DISCUSIÓN}

Durante el estudio morfológico y taxonómico de Eretmocerus, se observó que entre las características más importantes para la identificación de especies se encuentran las de las antenas, la longitud relativa de los segmentos antenales y la proporción entre la maza antenal, el ovipositor y la tibia media. Al considerar las descripciones de 
las especies mexicanas ya registradas, se encontró que cinco especies tienen la maza antenal más larga (7.1-9.1 veces tan larga como ancha), siete especies con longitud moderada (7.1-9.0 veces tan larga como ancha) y solamente tres especies contaban con la maza antenal corta (3.5-4.3 veces tan larga como ancha).

Además, se observó que la maza antenal más larga la tenían los parasitoides de Bemisia tabaci: de las siete especies con ese tipo de maza (más de 7 veces tan larga como ancha), cinco parasitan a dicha plaga. Por su parte, de las seis especies que atacan a Aleurothrixus floccosus, cuatro tienen la maza menor de 4.5 veces tan larga como ancha y aún tres de ellas presentan la maza más corta (3.5-4.3 veces tan larga como ancha). Además, estas tres especies tienen el pedicelo corto (1.8-2.2 veces tan largo como ancho). En comparación, las especies con maza antenal larga también tienen el pedicelo largo (3-4 veces tan largo como ancho), por ejemplo, Eretmocerus eremicus Rose \& Zolnerowich, E. tejanus Rose \& Zolnerowich y E. staufferi Rose \& Zolnerowich, parasitoides de Bemisia tabaci (ver también la Fig. 1 - antena de E. antennator Myartseva).

Por otra parte, se recibió para identificación material de Eretmocerus colectado con red entomológica en pastos por el Dr. Alejandro González-Hernández y colaboradores en el Estado de Jalisco, México. Dentro de este material se encontró la cuarta especie de Eretmocerus de México con maza antenal corta (3.6 veces tan larga como ancha) y se encontró que era nueva para la ciencia. Es posible que sea parasitoide de Aleurothrixus floccosus por tener la maza antenal corta.

\section{Revisión de las especies mexicanas de Eretmocerus con maza antenal corta} 1. Eretmocerus clavator Myartseva, sp. nov. (Fig. 2).

Descripción. Longitud del espécimen montado en bálsamo de Canadá - $0.55 \mathrm{~mm}$ ( $\sin$ contar el ovipositor).

Coloración. Cabeza y cuerpo amarillos, patas amarillo claro, ojos pardos, alas anteriores hialinas.

Estructura. Cabeza en vista frontal aprox. 1.5 veces más ancha que alta. Anchura del frontovértice ligeramente más de 0.5 la anchura de la cabeza. Ocelos posteriores separados del margen ocular por un poco más de un diámetro ocelar. Ojos aprox. 0.9 veces la longitud de las mejillas. Antenas insertadas debajo del nivel del margen inferior de los ojos. Radícula igual en longitud al pedicelo y 0.6 veces la longitud del escapo. Escapo 3 veces tan largo como ancho y 0.6 veces la longitud de la maza antenal. Pedicelo 2.2 veces tan largo como ancho. Primer segmento funicular triangular, segundo segmento ligeramente transversal. Maza antenal 3.6 veces tan larga como ancha, más bien ensanchada hacia el ápice; su anchura basal 0.4 veces la anchura máxima de la maza. Lóbulo medio del mesoescudo con seis setas, axila con una seta, lóbulo lateral con dos setas. Ala anterior 2.2 veces tan larga como la anchura alar 


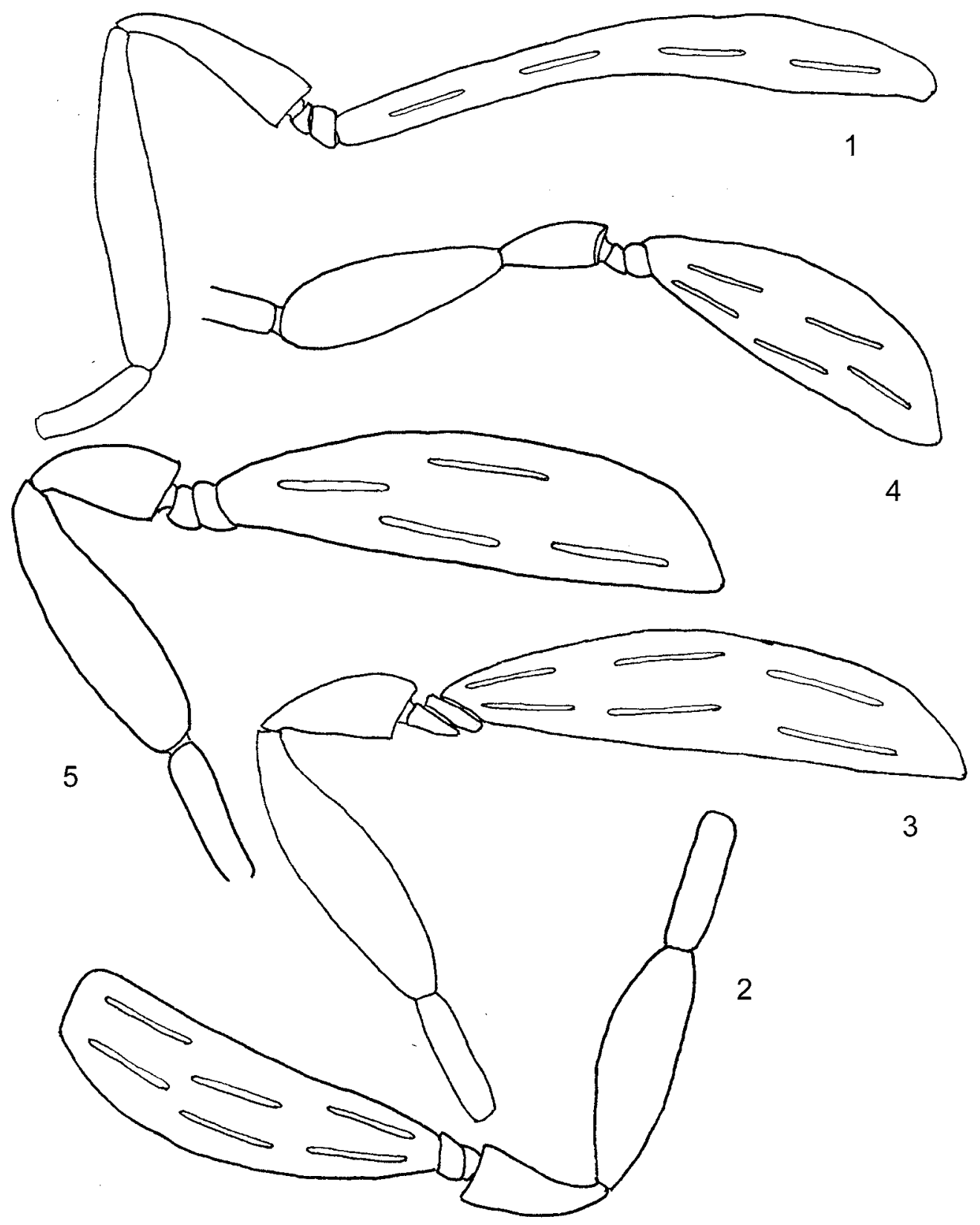

Figuras 1-5. Antenas de las hembras de Eretmocerus: $1-$ E. antennator, 2 - E. clavator, 3 -E. jimenezi, $4-E$. paulistus, 5 -E. portoricensis. 
máxima, fleco marginal aprox. 0.3 veces la anchura alar. Vena estigmal 1.5 veces más corta que la vena marginal. Nueve setas entre la vena marginal y la línea calva, 1-2 setas en la parte basal del disco. Línea calva cerrada posteriormente por unas pocas setas y tubérculos. Ala posterior 7.2 veces tan larga como ancha, su fleco marginal más o menos tan largo como la anchura alar máxima. Ovipositor fuertemente expuesto (la parte saliente aprox. 0.4 veces la longitud del ovipositor), cerca de 1.6 veces más larga que la tibia media y la maza antenal, las cuales son subiguales en longitud.

Macho desconocido.

Hospederos. Desconocidos.

Distribución. México (Estado de Jalisco).

Material examinado. Holotipo hembra: México, Jalisco, La Huerta, Est. Biol. Chamela UNAM, Vereda Tejón, redeo en maleza, 62 msnm, N 19³0'559', W 10502'464', 27.VII.2000 (col. Y. Castillo O., A. Dávila L. y A. González H.) (CIB 00-0039).

Diagnosis. La hembra de Eretmocerus clavator sp. nov. se distingue por la forma de la maza antenal, la cual es más bien ensanchada hacia el ápice y 3.6 veces tan larga como ancha, escapo 3 veces tan largo como ancho y 0.6 veces la longitud de la maza antenal, lóbulo medio del mesoescudo con 6 setas y ovipositor 1.6 veces tan largo como la tibia media y la maza antenal.

Es similar a E. portoricensis Dozier pero la maza de éste no se ensancha hacia el ápice y la longitud de su ovipositor es mayor (1.2) a la de la maza. E. joeballi Rose \& Zolnerowich y E. breviclavus Subba Rao también son similares a E. clavator pero en $E$. joeballi la maza antenal es 4.5-5.1 veces tan larga como ancha, escapo 4.5 veces tan largo como ancho, ovipositor 1.3 veces la longitud de la tibia media y 2.3 veces la longitud del escapo. En E. breviclavus la maza antenal es corta, aprox. 4.0 veces tan larga como ancha pero el escapo es 0.73 veces la longitud de la maza y el ovipositor es casi tan largo como la tibia media. E. longiterebrus Rose y E. paulistus Hempel también tienen ovipositor más largo que la maza y que la tibia media, las cuales son subiguales en longitud pero en E. clavator el ovipositor es 1.6 veces tan largo como la maza y la tibia media mientras que el ovipositor de $E$. longiterebrus es 1.26 veces tan largo como la maza y la tibia media, y en E. paulistus la relación es de 1.25 veces.

\section{Eretmocerus jimenezi Rose (Fig. 3)}

Eretmocerus jimenezi Rose, 2000: 26-29, Figs. 3, 23-24.

Descrita de México, holotipo hembra en el United States National Museum of Natural History (USNM). 
Hospedero. Aleurothrixus floccosus (Maskell).

Distribución. México (Colima, Guerrero, Michoacán, Nayarit, Nuevo León, San Luis Potosí, Sinaloa, Tamaulipas y Veracruz), El Salvador.

Diagnosis. Maza antenal aproximadamente 4.3 veces tan larga como ancha, escapo cerca de 3.8 veces tan largo como ancho, pedicelo aprox. 2.1 veces tan largo como ancho, primer segmento funicular antenal en triángulo recto elongado, segundo segmento reducido, muy comprimido y en forma de luna creciente; lóbulo medio del mesoescudo con 6 setas; ala anterior aprox. 2.5 veces tan larga como la anchura máxima, su fleco marginal aprox. 1/5 de la anchura del disco, de 9-11 setas entre la vena marginal y la línea calva; longitud de la espuela de la tibia media/ basitarso aprox. 1.0:1.6; longitud relativa de la maza antenal con el ovipositor y la tibia media 1.0:1.2, $1.0: 1.25$, respectivamente.

\section{Eretmocerus paulistus Hempel (Fig. 4)}

Eretmocerus paulistus Hempel, 1904: 19.

Descrita de Brasil, especímenes tipo perdidos; neotipo no designado ya que existe la posibilidad de que se encuentre el material de Hempel (Rose 2000). Redescripciones: Dozier 1932: 117; Rose 2000: 32-34, Figs. 27-28.

Hospederos. Aleyrodes horridus Hempel, Aleurothrixus floccosus, Neomaskellia bergii, Bemisia sp.

Distribución. Estados Unidos (California), México, Cuba, Haití, Perú, Brasil, Argentina, Chile, España.

Diagnosis. Lóbulo medio del mesoescudo con 4 setas; maza antenal ampliamente espatulada, 3.5 veces tan larga como ancha, escapo aprox. 3.5 veces tan largo como ancho, pedicelo cerca de 1.8 veces tan largo como ancho, primer segmento funicular en forma de triángulo reducido, segundo segmento comprimido y transversal; ala anterior aprox. 2.4 veces tan larga como ancha, fleco marginal cerca de $1 / 4$ de la anchura alar; de 9-12 setas entre la vena marginal y la línea calva; longitud de la espuela de la tibia media/ basitarso aprox. 1.0:1.4; longitud relativa de la maza antenal con el ovipositor y la tibia media 1.0:1.25, 1.0:1.05, respectivamente.

\section{Eretmocerus portoricensis Dozier (Fig. 5)}

Eretmocerus portoricensis Dozier, 1932: 115.

Descrita de Puerto Rico; lectotipo hembra en USNM, designado por Rose y Zolnerowich, 1997: 18; redescrita por Rose, 2000: 34-36, Figs. 29-31. 
Hospederos. Aleurothrixus floccosus, Tetraleurodes acaciae (Quaintance).

Distribución. Estados Unidos (Florida), México, Puerto Rico, República Dominicana, Perú.

Diagnosis. Maza antenal con ápice curvo, fuertemente convexo dorsalmente y recto ventralmente, 3.2-3.8 veces tan larga como ancha, escapo 3.5 veces tan largo como ancho, pedicelo 2.2 veces tan largo como ancho, igual a la radícula; primer segmento funicular triangular, tan ancho como largo, segundo segmento 1.6 veces tan ancho como largo; lóbulo medio del mesoescudo con 6 setas; ala anterior aprox. 2.7 veces tan larga como ancha, su fleco marginal aprox. 0.2 veces la anchura alar; de 10-13 setas entre la vena marginal y la línea calva; longitud de la espuela de la tibia media/ basitarso aprox. 1.0:1.6; ovipositor 1.2 veces tan largo como la maza antenal y de igual longitud que la tibia media.

\section{Clave para hembras de las especies mexicanas de Eretmocerus con maza antenal corta}

1. Lóbulo medio del mesoescudo con 4 setas. Maza antenal espatulada, 3.5 veces tan larga como ancha, escapo aprox. 3.5 veces tan largo como ancho, pedicelo aprox. 1.8 veces tan largo como ancho...................................paulistus Hempel

- Lóbulo medio del mesoescudo con 6 setas..................................................2

2. Primer segmento funicular antenal en ángulo recto con la base elongada, segundo segmento comprimido y en forma de luna creciente. Maza antenal aprox. 4.3 veces tan larga como ancha, escapo aprox. 3.8 veces tan largo como ancho, pedicelo aprox. 2.1 veces tan largo como ancho ........................jimenezi Rose

- Primer segmento funicular triangular, segundo segmento subcuadrado ...........3

3. Ápice de la maza antenal con un punto ancho; escapo 3.5 veces tan largo como ancho, ovipositor 1.2 veces tan largo como la maza ante-

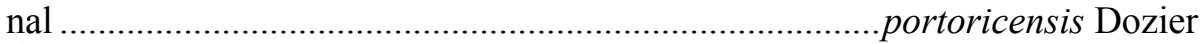

- Ápice de la maza antenal truncado; escapo 3 veces tan largo como ancho, ovipositor 1.6 veces tan largo como la maza antenal clavator Myartseva, sp. nov.

AGRADECIMIENTOS. Al Dr. Alejandro González-Hernández (Facultad de Ciencias Biológicas, Universidad Autónoma de Nuevo León, San Nicolás de Los Garza, Nuevo León, México) por el préstamo de su interesante colección de Eretmocerus. Al proyecto PROMEP "Taxonomía y ecología de fauna y micobiota en comunidades forestales y cultivos de México" de la Red de Cuerpos Académicos PROMEP y a la UAT, por su apoyo para la realización de este trabajo. 
Myartseva et al.: Eretmocerus de México con maza antenal corta

\section{LITERATURA CITADA}

Dozier, H.L. 1932. The identity of certain whitefly parasites of the genus Eretmocerus Hald., with descriptions of new species (Hymenoptera: Aphelinidae). Proceedings of the Entomological Society of Washington, 34: 112-122.

Hayat, M. 1998. Aphelinidae of India (Hymenoptera: Chalcidoidea): a taxonomic revision. Memoirs on Entomology, International, 13. 416 pp.

Myartseva, S. N. 2006. Eretmocerus Haldeman (Hymenoptera: Aphelinidae) - parasitoids of whiteflies Trialeurodes vaporariorum and Bemisia (tabaci complex) in Mexico, with a key and description of a new species. Vedalia, 13 (1): 27-38.

Myartseva, S. N. \& J. M. Coronado-Blanco. 2007. Especies de Eretmocerus Haldeman (Hymenoptera: Aphelinidae) - parasitoides de Aleurothrixus floccosus (Maskell) (Homoptera: Aleyrodidae) de México, con la descripción de una nueva especie. Acta Zoológica Mexicana, 23 (1): 37-46.

Noyes, J. S. 1982. Collecting and preserving chalcid wasps (Hymenoptera: Chalcidoidea). Journal of Natural History, 16: 315-334.

Noyes, J. S. 2003. Universal Chalcidoidea Database [online]. www.nhm.ac.uk/entomology/chalcidoids/index.html (última actualización: octubre 2010).

Rose, M. 2000. Eretmocerus Haldeman (Hymenoptera: Aphelinidae) reared from Aleurothrixus floccosus (Maskell) (Homoptera: Aleyrodidae) in the Americas. Vedalia, 7 (1): 3-46.

Rose, M. \& G. Zolnerowich. 1997. Eretmocerus Haldeman (Hymenoptera: Aphelinidae) in the United States, with descriptions of new species attacking Bemisia (tabaci complex) (Homoptera: Aleyrodidae). Proceedings of the Entomological Society of Washington, 99 (1): 1-27.

Zolnerowich, G. \& M. Rose. 1998. Eretmocerus Haldeman (Hymenoptera: Aphelinidae) imported and released in the United States for control of Bemisia (tabaci complex) (Homoptera: Aleyrodidae). Proceedings of the Entomological Society of Washington, 100 (2): 310-323. 